\title{
Post-Marketing Safety Surveillance for the Adjuvanted Recombinant Zoster Vaccine: Methodology
}

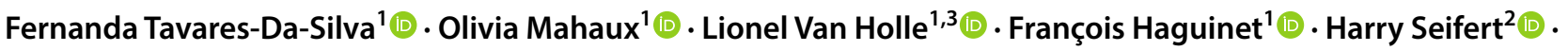 \\ Jens-Ulrich Stegmann ${ }^{1}$ (i)
}

Published online: 30 August 2020

(c) The Author(s) 2020

\begin{abstract}
A diligent, systematic, regular review of aggregate safety data is essential, particularly early after vaccine introduction, as this is when safety signals not identified during clinical development may emerge. In October 2017, the US Centers for Disease Control and Prevention Advisory Committee on Immunization Practices recommended the adjuvanted recombinant zoster vaccine (RZV; Shingrix, GSK) as the preferred vaccine for preventing herpes zoster (HZ) and related complications in immunocompetent adults aged $\geq 50$ years. Subsequently, GSK experienced an unprecedented high demand for RZV. In this methodology paper, we summarize the enhanced measures undertaken to assess RZV safety during its early post-marketing experience in the USA, Canada and Germany. In addition to the routine signal-detection methods already in place for all vaccines, GSK established tailored and enhanced safety monitoring for RZV based on aggregate data of spontaneous reports and manufacturing data. Proactive, near real-time detection and evaluation of signals was a key objective. A dedicated in-house signal-detection tool customized for RZV was employed on a weekly (rather than the routine monthly) basis, allowing for a centralized, more frequent review of data on a single web-based platform. We also identified the background incidence rates of preselected medical events of interest in the first countries to introduce RZV (USA, Canada and Germany) to perform observed-to-expected analyses. This approach may offer a solution to the challenges associated with the assessment and monitoring of vaccine safety in an efficient and timely manner in the context of high vaccine uptake.
\end{abstract}

Enhanced Digital Features To view enhanced digital features for this article, go to: https://doi.org/10.6084/m9.figshare.12730937.

Electronic supplementary material The online version of this article (https://doi.org/10.1007/s40264-020-00989-2) contains supplementary material, which is available to authorized users.

Fernanda Tavares-Da-Silva

fernanda.tavares@gsk.com

Olivia Mahaux

olivia.x.mahaux@gsk.com

Lionel Van Holle

lionel.vanholle@gmail.com

François Haguinet

francois.f.haguinet@gsk.com

Harry Seifert

Harry.A.Seifert@gsk.com

Jens-Ulrich Stegmann

jens-ulrich.x.stegmann@gsk.com

GSK, Avenue Fleming 20, 1300 Wavre, Belgium

2 GSK, 5 Crescent Drive, Philadelphia, PA, USA

3 Present Address: UCB Pharma, 1420 Braine-l'Alleud, Belgium

\section{Introduction}

Herpes zoster (HZ), commonly known as shingles, each year affects approximately 130,000 people in Canada [1], 1 million people in the USA [2], and 1.7 million in Europe [3]. It is estimated that around $20-30 \%$ of people will develop shingles in their lifetime [4].

Shingrix is an adjuvanted recombinant zoster vaccine (RZV) developed by GSK for the prevention of HZ in adults aged $\geq 50$ years. It is a subunit vaccine that contains recombinant varicella zoster virus glycoprotein $\mathrm{E}$ in combination with the adjuvant system $\mathrm{ASO1}_{\mathrm{B}}$ [5]. It is administered intramuscularly as two doses given 2-6 months apart. RZV has demonstrated over $90 \%$ efficacy against $\mathrm{HZ}$ across all age groups studied over 4 years of follow-up [5] and has a favourable benefit-risk profile [6-8].

In October 2017, RZV first received marketing authorization in Canada [9] and in the USA [10], and it is now also licensed in Europe (March 2018) [11], Japan (March 2018) [11], Australia (July 2018) [12] and China (May 2019) [13]. RZV is recommended by the US Centers for Disease Control and Prevention (CDC) Advisory Committee 


\section{Key Points}

The adjuvanted recombinant zoster vaccine (RZV) for prevention of herpes zoster in adults aged $\geq 50$ years was first licensed in the USA and Canada in 2017 and in Germany in 2018.

Given the novelty of this adjuvanted vaccine, which is used in the older adult population, a high volume of spontaneous reports was anticipated, particularly in the early uptake period post-licensure, which posed potential challenges for the rapid safety monitoring, detection and analysis of signals associated with RZV vaccination.

GSK developed a customized tool for the enhanced, near real-time safety surveillance of RZV during its first year of market introduction and performed a weekly evaluation of spontaneous report data on a single web-based platform.

We identified background rates of preselected medical events that could possibly occur in coincident temporal association with RZV vaccination, considering the older adult population targeted by the vaccine, to support signal detection as well as characterization activities, such as the observed-to-expected analyses.

Two examples of detection and/or earlier evaluation of signals using the methods described are provided in this article.

on Immunization Practices as the preferred vaccine for the prevention of $\mathrm{HZ}$ and related complications in immunocompetent adults aged $\geq 50$ years, over the previously available live-attenuated HZ vaccine (ZVL, Zostavax, Merck), because of RZV's higher and longer-lasting efficacy in older adults in separate clinical trials [14]. The Standing Committee on Vaccination (STIKO, Germany) also recommends RZV for the prevention of $\mathrm{HZ}$ in adults aged $\geq 60$ years and adults aged $\geq 50$ years with immunocompromising conditions or with severe underlying diseases [15]. In Canada, the National Advisory Committee on Immunization recommends the use of RZV to prevent HZ in adults aged $\geq 50$ years and that it may be considered on a case-by-case basis in immunocompromised adults aged $\geq 50$ years [16].

GSK collects spontaneous reports of adverse events (AEs) following immunization (AEFI) about all its vaccines. Spontaneous report data are collated from unsolicited communications describing one or more AEs that occur in patients who were given the vaccine. These communications, which are referred to as 'spontaneous reports', are either submitted to GSK directly and voluntarily from individual reporters via local reception/call centres or are collected by GSK from the scientific literature or the interactive digital media. The individual reporters can include healthcare professionals, regulatory authorities, consumers and others, who may be reporting for themselves or others. The rapid collection and evaluation of safety data received during the early period of introduction of a new vaccine in large-scale populations is pivotal for the early detection and investigation of signals (especially rare events) potentially linked to vaccination and that may not have emerged during pre-licensure clinical trials. It is worth pointing out that, while these AEFI are coincidental, they do not necessarily indicate a causal relationship with vaccination.

Given the expected high vaccine uptake and the novelty of RZV (adjuvanted) used in an older adult population, increased reporting was anticipated, particularly in the early uptake period post-licensure, for coincidental events that would be reported in close temporal association to vaccination, even though these would also have occurred regardless of vaccination. This context posed potential challenges for the timely and thorough post-marketing safety assessment of RZV. Therefore, in addition to routine pharmacovigilance activities, we implemented enhanced safety surveillance of RZV prior to and during its first year of licensure in the USA, Canada and Germany to rapidly detect, analyse and respond to potential safety signals in near real time with further investigation and signal-management activities. Evaluation of the safety profile after the early introduction of RZV was consistent with that previously observed in pre-licensure clinical trials and is the subject of another publication [17].

This article summarizes the data sources and methodology underlying the enhanced safety surveillance measures put in place by GSK for RZV and is illustrated with examples.

\section{Data Sources Used by GSK for Recombinant Zoster Vaccine (RZV) Signal Detection}

All internal and external data sources reviewed for RZV signal detection are outlined in the following sections.

\subsection{Internal (GSK) Data Sources}

\subsubsection{GSK Spontaneous Report Data}

All AEs spontaneously reported to GSK and obtained from reviews of the published literature are coded in the GSK worldwide safety database using the International Conference on Harmonisation Medical Dictionary for Regulatory Activities (MedDRA ${ }^{\circledR}$ ) [18]. A single spontaneous report can contain information about more than one $\mathrm{AE}$ (e.g. one 
report can describe fever, headache and malaise in the same individual). Data for signal detection and analyses following RZV vaccination were extracted from the GSK safety database, as is commonly done in GSK for other vaccines.

\subsubsection{RZV Post-Marketing Exposure Data}

The real-time exposure data (including number of doses administered) are not directly available to GSK because the vaccinations occur in standard clinical practice. However, the monthly distribution of RZV in the relevant countries was used as a proxy for RZV exposure under several assumptions of vaccine uptake, including that the doses were rapidly used and not stockpiled after distribution and that two-dose vaccination schedules were completed. The data are retrieved from an in-house database containing information provided by the GSK local country subsidiaries.

\subsubsection{Data Related to Vaccine Batch(es)}

Technical complaints and RZV batch data are also reported spontaneously to GSK. Such complaints may be purely technical (e.g. an empty or broken vial) or may be associated with reported AEs. The latter, as well as batch data, are used to detect a potential quality issue due to manufacturing [19].

\subsection{External Data Sources}

Spontaneous AEFI data following RZV vaccination from external national public databases, the US Vaccine Adverse Event Reporting System (VAERS) and the Canada Vigilance Adverse Reaction Online Database (CVAROD), were also extracted for review. Data from EudraVigilance, the system for monitoring the safety of medicines from the European Medicines Agency (EMA), were not yet available at the time of RZV launch but were extracted upon availability from October 2018 onwards and only at the aggregate level (electronic reaction monitoring reports [eRMRs]). These external data sources were selected from the countries where RZV is marketed (USA, Canada, Germany).

\subsubsection{US Spontaneous Report Data (VAERS)}

VAERS is a national early warning system to detect possible safety problems in US-licensed vaccines co-managed by the CDC and the US FDA and has been in operation since 1990 [20]. VAERS accepts and collects reports of post-vaccination AEs, and anyone can report AEs to VAERS.

De-identified VAERS data are available to the public approximately 4-6 weeks after they are reported and are readily accessible online for download [21]. GSK extracted the VAERS data related to RZV spontaneous reports monthly or upon availability.

\subsubsection{Canadian Spontaneous Report Data}

CVAROD contains information submitted to Health Canada about suspected adverse reactions to health products [22]. Adverse reaction reports can be voluntarily submitted by consumers and healthcare professionals. Manufacturers and distributors, also known as market authorization holders (MAHs), are required to submit reports according to the Canadian Food and Drugs Act.

Data were made available to the public by Health Canada on a quarterly basis until November 2018 and monthly thereafter [23]. The time lag between submission of a report and availability is 3-4 months. GSK extracted data related to RZV spontaneous reports from CVAROD as soon as they became available.

\subsubsection{European Spontaneous Report Data (EudraVigilance)}

As part of new reporting requirements in Europe, MAHs of certain active substances are required to monitor information on suspected adverse reactions in the new EMA EudraVigilance database during a transitional pilot period, which started on 22 February 2018, and to inform the EMA and national competent authorities of validated safety signals relating to their medicines. Although RZV was not part of the EudraVigilance pilot, RZV-related data have been made accessible to the public via EudraVigilance [24] and were considered as an additional source of new safety data. Therefore, GSK extracted eRMRs monthly once the active substance (higher level) name specific for RZV (i.e. recombinant varicella-zoster virus glycoprotein E) was determined, from October 2018 onwards.

\subsubsection{Observational Healthcare Data from the USA}

US claims databases such as the Truven Health MarketScan Commercial Database (Commercial Claims and Encounters [CCAE]) and the Medicare Supplemental Database (MDCR) were used to validate estimations of RZV exposure and distribution of exposure by age. This information was used to support observed-to-expected (O/E) analyses. The MarketScan CCAE contains healthcare data for patients with employer-based health insurance and their dependents who are primarily aged $<65$ years [25]. It has a broad geographic coverage and is an accurate representation of this population. The MDCR captures information on a subset of Medicare beneficiaries (primarily aged $\geq 65$ years) who possess supplemental insurance paid by their employers. Although the MDCR does not include data for all Medicare beneficiaries, it gives a fairly complete account of healthcare coverage patterns for those who are included in the database. 


\section{Signal Detection Methods}

\subsection{Quantitative Signal Detection}

\subsubsection{Routine Signal Detection}

GSK routinely performs quantitative signal detection for all marketed vaccines. A centralized review of data from multiple sources, including internal (GSK) and external data sources, is performed in a single web-based platform, the Signal Mining and Management (SMM) tool. These quantitative signals, along with relevant visualizations, are made available in SMM. SMM uses the GSK spontaneous report data, the GSK sales data and the EudraVigilance data for the products in scope of the pilot as raw data sources. Data are refreshed monthly for all vaccines.

Different algorithms and visualizations are built into the web application to analyse the data, which includes mining the raw data and signals, looking at trends, testing hypotheses, reviewing clinical details of cases, etc. Vaccine-event $(\mathrm{V}-\mathrm{E})$ pairs are flagged as quantitative signals if there is disproportionate reporting or evidence of an unexpected time-to-onset (TTO; the time between vaccination and the onset of the AE) distribution [26].

- A quantitative signal of disproportionate reporting for a $\mathrm{V}-\mathrm{E}$ pair is generated when the lower limit of the $95 \%$ confidence interval (CI) of the stratified proportional reporting ratio (PRR) is above the threshold of 2 . The PRR is stratified by sex, age group, geographic region and reporting period (13 October 2017-12 October 2019) when at least three cases are available per category.

- A quantitative signal of unexpected temporal relationship for a V-E pair is generated when its TTO distribution within 60 days post-vaccination is significantly different from the reported TTO distribution of that event of interest for other vaccines or from the reported TTO distribution of other events after the vaccine of interest. The chosen significance level is $1 \%(p<0.01)$ and the two-sample Kolmogorov-Smirnov (KS) test is used [26].

\subsubsection{Enhanced Safety Monitoring During the First Year of RZV Licensure}

For the first year of post-marketing surveillance of RZV, GSK reviewed spontaneous report aggregate data extracted from the safety database on a weekly basis rather than monthly, via the in-house Spotfire web application customized for RZV, using a new and unique approach to analyse the safety of RZV in near real time.

For this purpose, a global platform was established using a combination of software (SAS and Spotfire) to automate the data extraction from the aforementioned databases (including the GSK safety database, GSK sales data, GSK manufacturing database for the technical complaints, VAERS, CVAROD and EudraVigilance). These data from multiple sources, previously spread over different systems, were extracted and centralized for review into this single, customized web-based platform. We used two data-mining algorithms to highlight quantitative signals in an automated fashion: the disproportionality analyses and the KS twosample test for performing quantitative signal detection based on TTO as a complementary method [27]. Custom and embedded visualizations allow medical review of data in multiple ways and will save a significant amount of time. Searches can be performed to identify cases of interest or AEs of special interest (AESIs). The groups of AESIs can be searched using standard or customized MedDRA ${ }^{\circledR}$ Queries or using MedDRA ${ }^{\circledR}$ hierarchies. The automation and centralization of data allows fast and diligent recognition of signals and relevant communication to internal and/or external stakeholders. In addition, and as previously mentioned, weekly rather than monthly monitoring was performed to accommodate the rapid and high uptake for RZV. Video 1 (available online) gives an overview of the tool as accessed by users at the time of weekly release.

Disproportionality analysis was also customized for RZV. Instead of performing a stratified PRR analysis, as is done for routine analyses, a subset analysis was performed in which the background was restricted to a population aged $\geq 50$ years (the target population) and from countries in which RZV was marketed.

During the first year post-licensure, no safety concerns were identified, and data reinforced the clinically acceptable safety profile of RZV [17]. Therefore, the post-marketing surveillance for RZV continued using the routine monthly signal detection activities.

In addition to the enhanced safety monitoring for RZV, background incidence rates (IRs) were also estimated for medical conditions of interest, considering the target population for RZV. These additional analyses were not included in the in-house customized tool but prepared as a separate set of analyses.

\subsubsection{An Example of a Signal Detected for Vaccination Errors}

The first spontaneous reports for $\mathrm{RZV}$ were received in December 2017. In January 2018, a safety signal was identified due to the high percentage of reports $(52 \%$ of all reports received worldwide, $70 \%$ of all reports received from the USA) describing vaccination errors (mainly wrong route of administration, wrong reconstitution of the vaccine, or wrong storage conditions). Prior experience with other vaccines indicates that reports of vaccination errors are highest 
in the period shortly after launch $[28,29]$. Lack of familiarity with RZV and 10 years of previous use of ZVL in the USA [30] likely contributed to these errors. For example, ZVL should be stored in the freezer, reconstituted with a diluent and administered subcutaneously, which contrasts with RZV, which should be refrigerated, reconstituted with the supplied adjuvant $\mathrm{ASO1}_{\mathrm{B}}$ and administered intramuscularly. No clinical outcomes impacting the benefit-risk profile of RZV were identified during the case review, and the rapid signal identification and safety monitoring of vaccination errors offered a mechanism to introduce corrective strategies (such as implementation of educational programmes and product label information clarification). The tool has also allowed the continuous monitoring of cases of 'vaccination errors' through routine pharmacovigilance activities and follow-up on the implemented measures. With this, we have observed that vaccination errors involving reconstitution, storage and subcutaneous administration of RZV have decreased. In the USA, the percentage of vaccination errors decreased from 70\% in January 2018 to $25 \%$ in June 2018 [17].

An example of an overview of data and visualization of the vaccination error signal is shown in Video 1 (available online), and the display of the evolution of the quantitative signal over time in the web-based tool is presented in Fig. 2 in the electronic supplementary material (ESM).

\subsection{Signal Strengthening and Evaluation Methods}

\subsubsection{Background Incidence Rates for Medical Conditions of Interest}

Rates of background incidence are the number of incident cases of a disease or event occurring in a population prior to introduction of a new health intervention, expressed in person-time. Regulatory authorities generally recommend the integration of estimates of background IRs of AEs/ AESIs prior to vaccine introduction as part of routine pharmacovigilance practices, as these are essential to understand observed rates of AEs in the post-marketing setting [31-33].

GSK has built a repository of background IRs from the literature for selected medical conditions in target populations for RZV (i.e. adults aged $\geq 50$ years in the USA, Canada and Germany/Europe) (Table 1 in the ESM). This repository is updated by literature searches each time new analyses are considered.

These selected medical conditions included events of general interest for the assessment of vaccine safety and that could possibly occur in coincident temporal association with RZV vaccination, considering the novelty of the vaccine (adjuvanted) used in an older adult population, such as allcause mortality as well as a subset from the diverse group of potential immune-mediated diseases (pIMDs), which are events of special interest for adjuvanted vaccines [34]. The subset of pIMDs included psoriasis, rheumatoid arthritis, polymyalgia rheumatica, idiopathic thrombocytopenic purpura, small-vessel type vasculitis, multiple sclerosis, Guillain-Barré syndrome (GBS), temporal arteritis and Bell's palsy. Because the background IRs of these events are known to vary by age, country and over time, the background IRs in Supplementary Table 1 (see the ESM) were selected because they were recent, age-stratified and obtained from literature sources considered representative of the RZV-vaccinated populations. When background IRs were not available for a country of interest, we used background IRs from countries with similar characteristics, (e.g. data from Denmark extrapolated to Germany). For other vaccines, targeting a population with different characteristics, e.g. different age groups, ethnicity or sex, the background IRs considered relevant may differ from those presented here.

These background IRs are estimated from new onset/first diagnosis of the condition within a limited population during a certain follow-up period; these are not relevant to evaluate the potential influence of the vaccine on the frequency of relapses or exacerbations.

\subsubsection{Expected Number of Reports of Predefined Outcomes and Medical Events of Interest After Vaccination with RZV}

The number of cases of a specific medical event expected to occur by chance within a certain risk period is estimated based on background IRs for that event and total persontime at risk in the vaccinated population [31]. The expected number of events (Ne) after RZV vaccination adjusted by age was calculated using the following formula:

$N e=\sum_{s}[\text { Incidence rate }]_{s} \times[\text { Exposed persons }]_{s} \times$ time at risk

where incidence rate ${ }_{s}$, the incidence rate of the event in age stratum s; exposed persons, the number of individuals exposed in age stratum s; and time at risk, the time at risk accumulated per person depending on the risk period considered and the number of doses each person has received.

Table 1 presents the expected numbers of the predefined outcomes and medical events of interest after two-dose RZV vaccination for a generic exposed population of 100,000 individuals. If a potential safety signal was detected, O/E analyses were performed, determining whether the observed numbers of a reported AE were as expected within a predefined risk period, under the null hypothesis of no association between vaccination and onset of the event. Although $\mathrm{O} / \mathrm{E}$ analyses are signal-strengthening tools to support signal evaluation, we also incorporated the use of $\mathrm{O} / \mathrm{E}$ analyses earlier in the process for signal detection [31]. O/E analyses may provide additional insights during the review of 
aggregate data and can be complementary to routine signaldetection methods, particularly for AESIs. Nevertheless, this is only one method among other data sources and other quantitative methods available from the pharmacovigilance toolkit. O/E analyses are not suited to perform specific hypothesis testing or to measure the strength of associations between AEs and vaccines. The example in Sect. 3.2.3 presents a more in-depth $\mathrm{O} / \mathrm{E}$ analysis (including sensitivity analyses) for GBS.

\subsubsection{An Example of O/E Analysis for Guillain-Barré Syndrome}

An O/E analysis of spontaneous reports of GBS following RZV vaccination extracted from the GSK safety database was conducted for the analytical period 13 October 2017-12 October 2019. The O/E analysis was performed to assess whether the observed number of GBS reports corresponded to the number of events expected to occur within this predefined risk period.

All reports were reviewed based on Brighton Collaboration (BC) case definitions [35]. The $\mathrm{O} / \mathrm{E}$ analysis was performed considering $\mathrm{BC}$ levels 1-4 of diagnostics certainty. A case-control study demonstrated an increased risk of developing GBS in the 6 weeks after swine influenza vaccination [36], therefore a risk period of 42 days (day 0-41) following vaccination was used in this analysis. AE onset was used to assign cases to the respective risk windows. Cases with unknown AE onset were conservatively included in the analysis. Each dose was then considered as contributing independently to the total time at risk.

The exposure to RZV was estimated based on available sales data (doses distributed until 12 August 2019).

A literature search was performed to retrieve background IRs of GBS published in North America and Europe (countries where the majority of RZV doses were distributed during this analytic timeframe). The background IRs were standardized according to the age and sex distribution of the RZV recipients in each country. The age and sex distribution of the RZV recipients was approximated from all spontaneous reports of AEs following RZV vaccination recorded in the GSK safety database for which age at vaccination and sex was available.

Six references [32, 37-41] provided age- and sex-stratified estimates for North America and Europe, and the expected number of GBS events for these countries were able to be adjusted by age and sex. Two references [42, 43] only provided estimates stratified by age, so the corresponding expected numbers of GBS events were only adjusted by age. The paper by Benedetti et al. [37] presented the lowest estimate of background IRs, and the associated O/E ratio was the highest.
The observed number of cases and adjusted expected number of cases were computed globally. The reported number of cases and their Poisson exact 95\% CI were rescaled according to an arbitrary reported fraction (RF) of $75 \%$ to obtain the observed number of cases [44]. The RF is the proportion of cases reported among all those that occurred in the vaccinated population within the risk period, regardless of the causality.

The observed number of cases, along with the expected number of cases, considering a 42-day risk period and RF of $75 \%$, are shown in Table 2 in the ESM. The corresponding sensitivity analyses for varying levels of RFs and references are presented in Fig. 1 in the ESM, which represents the $\mathrm{O} / \mathrm{E}$ analyses conclusions considering different scenarios for background IRs and RF. Figure 1 in the ESM depicts a visual framework that enables independent reviewers to draw their conclusions by making their own assumptions about these two major sources of uncertainty. The observed cases received worldwide were below the expected number considering several scenarios of underreporting and background IRs. Only very low reporting rates would explain a higher observed incidence than expected.

\section{Discussion}

To prepare for the launch of RZV and the anticipated high number of spontaneous reports, GSK aimed to analyse the safety of RZV in near real time during the early post-marketing introduction. To achieve this, we developed a new, customized system for enhanced surveillance for RZV based on the use of an existing in-house tool.

One of the strengths of the GSK tool for enhanced surveillance is the centralized review of data from multiple sources in a single web-based platform, with relevant data mining algorithms and visualizations. This minimizes the risk of error, which may occur when switching between different systems, and the automation is time saving. This centralized overview also helps to create more meaningful statistics (e.g. the disproportionality analysis was fine tuned for RZV demographic specificities). Another advantage of the tool is that it allows for customization. The tool has been upgraded multiple times to integrate new data sources that require additional data extraction and manipulation outside of the tool (e.g. medical history), to add visualizations for specific events under close monitoring (e.g. reporting rates of medication errors over time), to facilitate identification of duplicate reports between the different database systems (VAERS, the GSK safety database, EudraVigilance) and to automate key data for communication to stakeholders. The system also allows for potential expansion of the scope of safety monitoring to other sources (e.g. claims data in the USA). The tool also offers great flexibility, allowing for 
Table 1 Expected number of predefined outcomes and medical events of interest after two-dose RZV vaccination, based on background incidence rates

\begin{tabular}{|c|c|c|c|c|c|}
\hline Country $^{\mathrm{a}}$ & $\begin{array}{l}\text { Risk } \\
\text { period } \\
\text { (year) }\end{array}$ & $\begin{array}{l}\text { Time at } \\
\text { risk }^{\mathrm{b}} \text { (year) }\end{array}$ & $\begin{array}{l}\text { Age-adjusted IR } \\
\text { (per 100,000 py) }\end{array}$ & $\begin{array}{l}\text { Expected number (per } \\
100,000 \text { vaccinated) }\end{array}$ & Reference for IR \\
\hline \multicolumn{6}{|l|}{ Psoriasis } \\
\hline USA & 0.49 & 0.66 & 82.3 & 54 & \multirow[t]{2}{*}{ Icen et al. [49] } \\
\hline Canada & 0.49 & 0.66 & 85.1 & 56 & \\
\hline Germany & 0.49 & 0.66 & 196.1 & 129 & Egeberg et al. [50] \\
\hline \multicolumn{6}{|c|}{ Rheumatoid arthritis } \\
\hline USA & 0.49 & 0.66 & 75.2 & 49 & \multirow[t]{2}{*}{ Myasoedova et al. [51] } \\
\hline Canada & 0.49 & 0.66 & 72.2 & 47 & \\
\hline Germany & 0.49 & 0.66 & 71.9 & 47 & Abhishek et al. [52] \\
\hline \multicolumn{6}{|c|}{ Polymyalgia rheumatica } \\
\hline USA & 0.49 & 0.66 & 80.5 & 53 & \multirow[t]{3}{*}{ Raheel et al. [53] } \\
\hline Canada & 0.49 & 0.66 & 59.1 & 39 & \\
\hline Germany & 0.49 & 0.66 & 56.9 & 37 & \\
\hline \multicolumn{6}{|c|}{ Idiopathic thrombocytopenic purpura } \\
\hline USA & 0.11 & 0.23 & 5.4 & 1 & \multirow[t]{3}{*}{ Schoonen et al. [54] } \\
\hline Canada & 0.11 & 0.23 & 4.8 & 1 & \\
\hline Germany & 0.11 & 0.23 & 4.5 & 1 & \\
\hline \multicolumn{6}{|c|}{ Cutaneous leukocytoclastic vasculitis } \\
\hline USA & 0.11 & 0.23 & 2.1 & 0 & \multirow[t]{2}{*}{ Arora et al. [55] } \\
\hline Canada & 0.11 & 0.23 & 2.1 & 0 & \\
\hline Germany & 0.11 & 0.23 & 3.02 & 1 & $\begin{array}{l}\text { Garcia-Porrua and Gonzalez- } \\
\text { Gay [56] }\end{array}$ \\
\hline \multicolumn{6}{|c|}{ Multiple sclerosis } \\
\hline USA & 0.49 & 0.66 & 3.3 & 2 & O'Connell et al. [57] \\
\hline Canada & 0.49 & 0.66 & 14.1 & 9 & Al-Sakran et al. [58] \\
\hline Germany & 0.49 & 0.66 & 4.6 & 3 & O'Connell et al. [57] \\
\hline \multicolumn{6}{|c|}{ Guillain-Barré syndrome } \\
\hline USA & 0.11 & 0.23 & 3.7 & 1 & Frenzen [42] \\
\hline Canada & 0.11 & 0.23 & 2.8 & 1 & Hauck et al. [39] \\
\hline Germany & 0.11 & 0.23 & 3.8 & 1 & Hense et al. [40] \\
\hline \multicolumn{6}{|c|}{ Temporal arteritis } \\
\hline USA & 0.16 & 0.33 & 23.6 & 8 & \multirow[t]{2}{*}{ Chandran et al. [59] } \\
\hline Canada & 0.16 & 0.33 & 16.2 & 5 & \\
\hline Germany & 0.16 & 0.33 & 3.7 & 1 & Reinhold-Keller et al. [60] \\
\hline \multicolumn{6}{|l|}{ Bell's palsy } \\
\hline USA & 0.08 & 0.16 & 40.8 & 7 & \multirow[t]{3}{*}{ Black et al. [32] } \\
\hline Canada & 0.08 & 0.16 & 39.3 & 6 & \\
\hline Germany & 0.08 & 0.16 & 37.6 & 6 & \\
\hline \multicolumn{6}{|c|}{ Mortality (all causes) } \\
\hline USA & 0.02 & 0.04 & 2153.7 & 83 & $\mathrm{CDC}[61]$ \\
\hline Canada & 0.02 & 0.04 & 1332.2 & 51 & STATCAN [62] \\
\hline Germany & 0.02 & 0.04 & 1066.4 & 41 & GBE [63] \\
\hline
\end{tabular}

$C D C$ Centers for Disease Control and Prevention, GBE Gesundheitsberichterstattung des Bundes, $I R$ incidence rates, py person-year, $R Z V$ recombinant zoster vaccine, STATCAN Statistics Canada

${ }^{a}$ Country for which expected number analysis was performed. When background IR data were unavailable or unreliable, data from countries judged to have similar characteristics were used as proxy

${ }^{\mathrm{b}}$ The time a person is at risk equals the cumulative risk period after each dose when the risk period is $<2$ months, 2 months being the time interval between two consecutive doses. When the risk period extends past 2 months, then the risk period after dose 1 temporally overlaps with the second dose and the cumulative time at risk for a person is 2 months after dose 1 , to avoid double counting risk periods, added to a complete risk period after dose 2

${ }^{c}$ In age-stratified analyses, each age stratum is provided a weight based on the age distribution of the exposed population. Therefore, the age-adjusted IR for individuals vaccinated with RZV has been estimated by taking the weighted average of the incidence rates within each age stratum 
weekly versus the routine monthly analysis. Although considerably more resources were needed in the preparedness phase, it added value to the routine safety monitoring methods because this tailored approach was relatively simple to implement using standard computer software and methods, assisting safety reviewers in analysing large sets of data, identifying sets of cases of potential interest and facilitating appropriate prioritization for further evaluation. Together, these features allowed rapid identification of and response to safety signals.

The enhanced surveillance tool uses input from different sources that mostly rely on spontaneous reporting (including the GSK safety database, VAERS, CVAROD and EudraVigilance). The main advantages of spontaneous reporting systems include the coverage of large populations and the ability to collect data on rare events that may be related to vaccination but are not always detected during clinical development. The collection of information from multiple spontaneous reporting sources for RZV allows a more complete collection of data from entire population sets and enables GSK to more rapidly detect possible safety issues.

However, spontaneous reporting suffers from several limitations $[45,46]$. In particular, a key problem is the lack of denominator data to rapidly estimate vaccine usage (number of people vaccinated) in the target population. The lack of denominators also presents a challenge when performing signal-strengthening activities, such as $\mathrm{O} / \mathrm{E}$ analyses [31], where the observed number of cases constituting the signal is compared with an expected number of cases estimated from IRs in a current or historical unexposed population. To overcome this, we used RZV sales data as a proxy for exposure. Reliable and near real-time data on actual usage of the vaccine (rather than sales or distribution data) in the relevant region(s) are essential for monitoring and analysis of potential safety signals but are not always available. Initiatives such as the VAC4EU (Vaccine monitoring Collaboration for Europe) within the ADVANCE project in Europe have been created to actively monitor vaccine coverage in near real time [47] and will be key to further improving the monitoring and analysis of safety signals arising from spontaneous report data. The rapid cycle analysis from the Vaccine Safety Datalink network in the USA is another example of a tool that performs monthly near real-time monitoring of vaccine safety.

Another challenge in analysing data simultaneously from three different spontaneous reporting systems is that some reports are present in the three systems, some in two systems and others only in one. Vogel et al. [48] reported this issue for other spontaneous reporting databases. The extent of this overlap between the spontaneous case reports from the different sources is unknown. A duplicate check was applied at data entry of the case report in the GSK safety database; however, it could be difficult to reconcile the same reports from the different databases because of the limited access to personal information. Some reports from other systems that are already present in the GSK database can be identified within the tool because they have a similar ID code corresponding to the GSK ID. Developing a standard duplicate-detection method in the SMM tool would allow easier identification of duplicates across different spontaneous reports, which in turn would permit a more robust estimation of the number of unique spontaneous reports observed across reporting systems when running the O/E analyses. Of note, the investigation and analysis of the external data related to RZV spontaneous reports by GSK may sometimes be impacted by the time lag between reporting and data availability in the external spontaneous reporting systems, such as VAERS. However, given that data are frequently refreshed/analysed, we do not believe this has a major impact on our analyses.

Background IRs can be used to estimate the number of events expected to occur in the absence of vaccination in the relevant population and to help determine the likelihood that the observed cases after vaccination occur only by chance or are true signals. For the calculations of the expected number of cases presented in Table 1, some assumptions were made: (1) all individuals were compliant, i.e. received two doses 2-6 months apart; (2) the reported age was representative of the actual age distribution in the exposed population; (3) there was no dose effect, meaning that the risk was considered identical after each dose.

These assumptions may not always be correct. In the MarketScan data available at the time of this assessment, the vast majority of people ( $>90 \%$ ) only received one dose (possibly because demand for RZV exceeded supply during the observation time), thereby reducing the expected number of cases in Table 1 by $25-50 \%$, depending on the risk period. Moreover, the expected numbers of cases in the risk period presented in Table 1 are impacted when the age distribution at RZV vaccination is estimated from the pooled MarketScan data instead of the spontaneous reports data. For temporal arteritis, for example, this would result in a 35\% reduction of the expected number presented in Table 1. Consequently, there may be an over- or underestimation of the significance of potential findings. In the example for temporal arteritis, a reduction in the number of expected cases could result in biased estimates. However, an important limitation of this approach is that each MarketScan data source may include non-matching specific socioeconomic parts of the US population in their respective age range. Therefore, the standardization of the data according to the predicted US population may also have its limitations, and the age distribution of the vaccinated population obtained from the pooled MarketScan data may not be a good proxy of the age distribution in the US exposed population. This demonstrates the importance of having actual vaccine exposure data stratified by age and 
reliable data on vaccination compliance with the recommended vaccination schedule.

Choosing the most relevant background IRs for vaccine safety evaluation in the context of the anticipated real-world use of RZV represents a challenge. Some countries will have no data available for background IRs for selected events of interest. As much as possible, the background IRs are chosen to limit biases and should be estimated from a population sharing the same demographic characteristics as the exposed population. One characteristic to take into consideration is the country or region, particularly for medical conditions influenced by genetic and environmental factors. Therefore, background IRs were chosen to correspond to countries/ regions exposed to RZV. Another factor is age because it can influence certain medical conditions. Background IRs therefore focused on a population aged $\geq 50$ years and were further stratified by age when this information was available. Background IRs were chosen to be as recent as possible given the available literature because the contemporaneity of the data is important in case of secular trends in the incidence of the medical condition, for example due to increased medical knowledge of the disease or improvement of diagnostic techniques or definitions. In case of incomplete matching of the above characteristics, the lowest IR estimates may be used as a worst-case approach for the evaluation of the vaccine safety, provided those are not outliers. The design and robustness of the method in epidemiological studies are also important points but, in this work, where the medical conditions were chosen a priori and standard definitions and case ascertainment process were not yet decided, less importance is given to those aspects of the method. Because not all the information needed for case ascertainment is always available in reports derived from passive surveillance, more importance was given to using definitions and case ascertainment processes for the background IRs that matched those that can be used for the spontaneously reported data that will give rise to a signal.

In our opinion, the success factors in terms of safety monitoring included the earlier implementation of a tailored signal detection and evaluation for RZV, and the near realtime analysis of data (weekly rather than monthly) using the centralized review of data from multiple sources in a single web-based platform. In addition, we used two data-mining algorithms and incorporated the use of $\mathrm{O} / \mathrm{E}$ analyses earlier in the process as a complementary method for routine signal detection.

The results from the analysis of RZV's safety profile during the first year of market introduction are discussed in another publication [17]. It would be of interest to continue formulating a unified, sequential approach in which vaccine safety monitoring systems and methods can be simplified and improved to potentially mitigate the impact of future vaccine safety controversies and to identify safety signals more quickly and accurately for better patient care. This paper aims to take that approach by sharing our experience with potential pre- and post-licensure opportunities to improve preparedness for vaccine safety surveillance, using the example of RZV. The same methodology is currently used for routine monitoring of other products as it allows for customization to the product needs, adding different data sources and potential future enhancements.

\section{Conclusions}

A proactive, more frequent analysis of spontaneous safety data was established for RZV in anticipation of large numbers of medical events-which need further investigation to identify true safety signals-for this newly licensed vaccine, given its preferential recommendation and defined medical needs. Our enhanced safety monitoring approach for RZV was based on spontaneous reports data. A dedicated in-house analytical tool customized for RZV was employed weekly within a single web-based platform. We used age-stratified IRs to determine how many medical events of interest would be expected following vaccination. This allowed near realtime evaluation of the likelihood of excess reporting after adjustment for underreporting.

We believe this approach could be adopted for other newly licensed vaccines with expected rapid uptake post-licensure in countries with well-established pharmacovigilance systems and with background IRs available for outcomes of interest, allowing for rapid identification of safety signals arising from spontaneous data as efficiently as possible.

Acknowledgements The authors thank Vincent Bauchau, David Willer, Carla Talarico, Anne Schuind and Jacqueline Miller for their critical review of the manuscript and thank Pascale Schrauben for her assistance during the development of the video. We also thank Kristel Vercauteren for writing assistance and Salomé Murinello (Modis, Wavre, Belgium), on behalf of GSK (Wavre, Belgium), for coordination and editorial support.

\section{Declarations}

Funding This study was funded by GlaxoSmithKline Biologicals SA. GlaxoSmithKline Biologicals SA also covered the costs associated with the development and publishing of the manuscript.

Conflicts of interest FTS, JUS, FH, HAS and OM are employees of the GSK group of companies and FTS, LVH, JUS, FH, HAS and OM hold shares/restricted shares in the GSK group of companies. LVH was employed by the GSK group of companies during the conduct of this study and is a current employee of UCB Pharma. Fernanda TavaresDa-Silva, Olivia Mahaux, Lionel Van Holle, François Haguinet, Harry Seifert and Jens-Ulrich Stegmann have no other conflicts of interest that are directly relevant to the content of this article.

Ethics approval Not applicable 
Consent to participate Not applicable

Consent for publication Not applicable

Availability of data and material The data that support the findings of this work are available from the corresponding author on reasonable request.

\section{Code availability Not applicable.}

Author contributions FTS, LVH, JUS and OM were involved in the conception and design of the study; HAS, FTS, LVH, JUS and OM contributed to the data extraction; FTS, FH and JUS conducted the analyses; and all authors contributed to interpreting the data. All authors reviewed and edited the manuscript. All authors have read and approved the final manuscript. All authors attest they meet the International Committee of Medical Journal Editors criteria for authorship.

Open Access This article is licensed under a Creative Commons Attribution-NonCommercial 4.0 International License, which permits any non-commercial use, sharing, adaptation, distribution and reproduction in any medium or format, as long as you give appropriate credit to the original author(s) and the source, provide a link to the Creative Commons licence, and indicate if changes were made. The images or other third party material in this article are included in the article's Creative Commons licence, unless indicated otherwise in a credit line to the material. If material is not included in the article's Creative Commons licence and your intended use is not permitted by statutory regulation or exceeds the permitted use, you will need to obtain permission directly from the copyright holder. To view a copy of this licence, visit http://creativecommons.org/licenses/by-nc/4.0/.

\section{References}

1. Brisson M, Pellissier JM, Camden S, Quach C, De Wals P. The potential cost-effectiveness of vaccination against herpes zoster and post-herpetic neuralgia. Hum Vaccin. 2008;4:238-45.

2. Harpaz R, Ortega-Sanchez IR, Seward JF, Advisory Committee on Immunization Practices Centers for Disease Control and Prevention. Prevention of herpes zoster: recommendations of the Advisory Committee on Immunization Practices (ACIP). MMWR Recomm Rep. 2008;57:1-30; quiz CE2-4.

3. Pinchinat S, Cebrian-Cuenca AM, Bricout H, Johnson RW. Similar herpes zoster incidence across Europe: results from a systematic literature review. BMC Infect Dis. 2013;13:170.

4. Zorzoli E, Pica F, Masetti G, Franco E, Volpi A, Gabutti G. Herpes zoster in frail elderly patients: prevalence, impact, management, and preventive strategies. Aging Clin Exp Res. 2018;30:693-702.

5. Lecrenier N, Beukelaers P, Colindres R, Curran D, De Kesel C, De Saegher JP, et al. Development of adjuvanted recombinant zoster vaccine and its implications for shingles prevention. Expert Rev Vaccines. 2018;17:619-34.

6. Cunningham AL, Lal H, Kovac M, Chlibek R, Hwang SJ, DiezDomingo J, et al. Efficacy of the herpes zoster subunit vaccine in adults 70 years of age or older. N Engl J Med. 2016;375:1019-32.

7. Lal H, Cunningham AL, Godeaux O, Chlibek R, Diez-Domingo J, Hwang SJ, et al. Efficacy of an adjuvanted herpes zoster subunit vaccine in older adults. Vaccine. 2020;38(18):3489-500.

8. Lopez-Fauqued M, Campora L, Delannois F, El Idrissi M, Oostvogels L, De Looze FJ, et al. Safety profile of the adjuvanted recombinant zoster vaccine: pooled analysis of two large randomised phase 3 trials. Vaccine. 2019;37:2482-93.
9. GSK. GSK announces first approval of Shingrix in Canada. 2020. https://www.gsk.com/en-gb/media/press-releases/gsk-announcesfirst-approval-of-shingrix-in-canada/. Accessed 13 Jul 2020.

10. GSK. Shingrix approved in the US for prevention of shingles in adults aged 50 and over. 2020. https://www.gsk.com/en-gb/ media/press-releases/shingrix-approved-in-the-us-for-prevention -of-shingles-in-adults-aged-50-and-over/. Accessed 13 Jul 2020.

11. GSK. Shingrix approved in Europe and Japan for the prevention of shingles in adults aged 50 and over. 2020. https://www.gsk.com/ en-gb/media/press-releases/shingrix-approved-in-europe-andjapan-for-the-prevention-of-shingles-in-adults-aged-50-and-over/. Accessed 13 Jul 2020.

12. Australian Government Department of Health TGA. Australian public assessment report for recombinant varicella zoster virus (VZV) glycoprotein E (gE) antigen. 2020. https://www.tga.gov.au/ sites/default/files/auspar-recombinant-varicella-zoster-virus-glyco protein-e-antigen-181212.pdf. Accessed 10 Jul 2020.

13. GSK. GSK announces approval of Shingrix in China for prevention of shingles in adults aged 50 and over. 2020. https://www.gsk. com/en-gb/media/press-releases/gsk-announces-approval-of-shing rix-in-china-for-prevention-of-shingles-in-adults-aged-50-andover/. Accessed 13 Jul 2020

14. Dooling KL, Guo A, Patel M, Lee GM, Moore K, Belongia EA, et al. Recommendations of the Advisory Committee on Immunization Practices for use of herpes zoster vaccines. MMWR Morb Mortal Wkly Rep. 2018;67:103-8.

15. Robert Koch Institut. Epidemiologisches bulletin. 2020. https ://www.rki.de/DE/Content/Infekt/EpidBull/Archiv/2018/Ausga ben/50_18.pdf?_blob=publicationFile. Accessed $10 \mathrm{Jul} 2020$.

16. Warrington $\mathrm{R}$, Ismail $\mathrm{S}$, National Advisory Committee on Immunization. Summary of the NACI update on herpes zoster vaccines. Can Commun Dis Rep. 2018;44:220-5.

17. Tavares-Da-Silva F, Miranda M, Dessart C, Herve C, LopezFauqued M, Mahaux O, et al. Review of the initial post-marketing safety surveillance for the recombinant zoster vaccine. Vaccine. 2019.

18. Medical Dictionary for Regulatory Activities (MedDRA). 2020. https://www.meddra.org/. Accessed 10 Jul 2020.

19. Mahaux O, Bauchau V, Zeinoun Z, Van Holle L. Tree-based scan statistic - Application in manufacturing-related safety signal detection. Vaccine. 2019;37:49-55.

20. U.S. Department of Health and Human Services. Vaccine Adverse Event Reporting System (VAERS). 2020. https://vaers.hhs.gov/. Accessed 10 Jul 2020.

21. U.S. Department of Health and Human Services. Vaccine Adverse Event Reporting System (VAERS): VAERS data. 2020. https:// vaers.hhs.gov/data/datasets.html. Accessed 10 Jul 2020.

22. Government of Canada. Canadian Vigilance Adverse Reaction Online Database. 2020. https://www.canada.ca/en/health-canad a/services/drugs-health-products/medeffect-canada/adverse-react ion-database.html. Accessed 10 Jul 2020.

23. Government of Canada. Data extracts from the Canada Vigilance adverse reaction online database. 2020. https://www.canada.ca/en/ health-canada/services/drugs-health-products/medeffect-canada/ adverse-reaction-database/canada-vigilance-online-database-dataextract.html. Accessed $10 \mathrm{Jul} 2020$.

24. EudraVigilance. European database of suspected adverse drug reaction reports. 2020. http://www.adrreports.eu/en/index.html. Accessed 10 Jul 2020.

25. Quint JB. Health Research data for the real world: the MarketScan databases. Ann Arbor: Truven Health Analytics; 2015.

26. Van Holle L, Zeinoun Z, Bauchau V, Verstraeten T. Using timeto-onset for detecting safety signals in spontaneous reports of adverse events following immunization: a proof of concept study. Pharmacoepidemiol Drug Saf. 2012;21:603-10. 
27. Van Holle L, Bauchau V. Use of logistic regression to combine two causality criteria for signal detection in vaccine spontaneous report data. Drug Saf. 2014;37:1047-57.

28. Hibbs BF, Moro PL, Lewis P, Miller ER, Shimabukuro TT. Vaccination errors reported to the Vaccine Adverse Event Reporting System, (VAERS) United States, 2000-2013. Vaccine. 2015;33:3171-8.

29. Su JR, Miller ER, Duffy J, Baer BM, Cano MV. Notes from the field: Administration error involving a meningococcal conjugate vaccine-United States, March 1, 2010-September 22, 2015. MMWR Morb Mortal Wkly Rep. 2016;65:161-2.

30. Zostavax (Zoster Vaccine Live). Highlights of prescribing information. 2020. http://www.fda.gov/media/119879/download. Accessed 14 Jun 2020.

31. Mahaux O, Bauchau V, Van Holle L. Pharmacoepidemiological considerations in observed-to-expected analyses for vaccines. Pharmacoepidemiol Drug Saf. 2016;25:215-22.

32. Black S, Eskola J, Siegrist CA, Halsey N, MacDonald N, Law B, et al. Importance of background rates of disease in assessment of vaccine safety during mass immunisation with pandemic H1N1 influenza vaccines. Lancet. 2009;374:2115-22.

33. Tavares Da Silva F, Di Pasquale A, Yarzabal JP, Garcon N. Safety assessment of adjuvanted vaccines: Methodological considerations. Hum Vaccin Immunother. 2015;11:1814-24.

34. Tavares Da Silva F, De Keyser F, Lambert PH, Robinson WH, Westhovens R, Sindic C. Optimal approaches to data collection and analysis of potential immune mediated disorders in clinical trials of new vaccines. Vaccine. 2013;31:1870-6.

35. Sejvar JJ, Kohl KS, Gidudu J, Amato A, Bakshi N, Baxter R, et al. Guillain-Barre syndrome and Fisher syndrome: case definitions and guidelines for collection, analysis, and presentation of immunization safety data. Vaccine. 2011;29:599-612.

36. Schonberger LB, Bregman DJ, Sullivan-Bolyai JZ, Keenlyside RA, Ziegler DW, Retailliau HF, et al. Guillain-Barre syndrome following vaccination in the National Influenza Immunization Program, United States, 1976-1977. Am J Epidemiol. 1979;110:105-23.

37. Benedetti MD, Pugliatti M, D’Alessandro R, Beghi E, Chio A, Logroscino G, et al. A Multicentric prospective incidence study of Guillain-Barre syndrome in Italy. The ITANG Study. Neuroepidemiology. 2015;45:90-9.

38. Delannoy A, Rudant J, Chaignot C, Bolgert F, Mikaeloff Y, Weill A. Guillain-Barre syndrome in France: a nationwide epidemiological analysis based on hospital discharge data (2008-2013). J Peripher Nerv Syst. 2017;22:51-8.

39. Hauck LJ, White C, Feasby TE, Zochodne DW, Svenson LW, Hill MD. Incidence of Guillain-Barre syndrome in Alberta, Canada: an administrative data study. J Neurol Neurosurg Psychiatry. 2008;79:318-20

40. Hense S, Schink T, Kreisel SH, Marcelon L, Simondon F, Tahden $\mathrm{M}$, et al. Estimation of background incidence rates of GuillainBarre syndrome in Germany - a retrospective cohort study with electronic healthcare data. Neuroepidemiology. 2014;43:244-52.

41. Skufca J, Ollgren J, Ruokokoski E, Lyytikainen O, Nohynek H. Incidence rates of Guillain Barre (GBS), chronic fatigue/systemic exertion intolerance disease (CFS/SEID) and postural orthostatic tachycardia syndrome (POTS) prior to introduction of human papilloma virus (HPV) vaccination among adolescent girls in Finland, 2002-2012. Papillomavirus Res. 2017;3:91-6.

42. Frenzen PD. Hospital admissions for Guillain-Barre syndrome in the United States, 1993-2004. Neuroepidemiology. 2007;29:83-8.

43. van der Maas NA, Kramer MA, Jacobs BC, van Soest EM, Dieleman JP, Kemmeren JM, et al. Guillain-Barre syndrome: background incidence rates in The Netherlands. J Peripher Nerv Syst. 2011;16:243-9.

44. Garwood F. (i) Fiducial limits for the Poisson distribution. Biometrika. 1936;28:437-42.

45. Goldman SA. Limitations and strengths of spontaneous reports data. Clin Ther. 1998;20 Suppl C:C40-4.

46. Shimabukuro TT, Nguyen M, Martin D, DeStefano F. Safety monitoring in the Vaccine Adverse Event Reporting System (VAERS). Vaccine. 2015;33:4398-405.

47. Vaccine monitoring collaboration for Europe. 2020. https://vac4e u.org/. Accessed 11 Jun 2020.

48. Vogel U, van Stekelenborg J, Dreyfus B, Garg A, Habib M, Hosain $\mathrm{R}$, et al. Investigating overlap in signals from EVDAS, FAERS, and VigiBase(®). Drug Saf. 2020;43:351-62.

49. Icen M, Crowson CS, McEvoy MT, Dann FJ, Gabriel SE, Maradit Kremers H. Trends in incidence of adult-onset psoriasis over three decades: a population-based study. J Am Acad Dermatol. 2009;60:394-401.

50. Egeberg A, Skov L, Gislason GH, Thyssen JP, Mallbris L. Incidence and prevalence of psoriasis in Denmark. Acta Derm Venereol. 2017;97:808-12.

51. Myasoedova E, Crowson CS, Kremers HM, Therneau TM, Gabriel SE. Is the incidence of rheumatoid arthritis rising?: results from Olmsted County, Minnesota, 1955-2007. Arthritis Rheum. 2010;62:1576-82.

52. Abhishek A, Doherty M, Kuo CF, Mallen CD, Zhang W, Grainge MJ. Rheumatoid arthritis is getting less frequent-results of a nationwide population-based cohort study. Rheumatology (Oxford). 2017;56:736-44.

53. Raheel S, Shbeeb I, Crowson CS, Matteson EL. Epidemiology of polymyalgia rheumatica 2000-2014 and examination of incidence and survival trends over 45 years: a population-based study. Arthritis Care Res (Hoboken). 2017;69:1282-5.

54. Schoonen WM, Kucera G, Coalson J, Li L, Rutstein M, Mowat F, et al. Epidemiology of immune thrombocytopenic purpura in the General Practice Research Database. Br J Haematol. 2009; 145:235-44.

55. Arora A, Wetter DA, Gonzalez-Santiago TM, Davis MD, Lohse CM. Incidence of leukocytoclastic vasculitis, 1996 to 2010: a population-based study in Olmsted County, Minnesota. Mayo Clin Proc. 2014;89:1515-24.

56. Garcia-Porrua C, Gonzalez-Gay MA. Comparative clinical and epidemiological study of hypersensitivity vasculitis versus Henoch-Schonlein purpura in adults. Semin Arthritis Rheum. 1999;28:404-12.

57. O'Connell K, Tubridy N, Hutchinson M, McGuigan C. Incidence of multiple sclerosis in the Republic of Ireland: a prospective population-based study. Mult Scler Relat Disord. 2017;13:75-80.

58. Al-Sakran LH, Marrie RA, Blackburn DF, Knox KB, Evans CD. Establishing the incidence and prevalence of multiple sclerosis in Saskatchewan. Can J Neurol Sci. 2018;45:295-303.

59. Chandran AK, Udayakumar PD, Crowson CS, Warrington KJ, Matteson EL. The incidence of giant cell arteritis in Olmsted County, Minnesota, over a 60-year period 1950-2009. Scand J Rheumatol. 2015;44:215-8.

60. Reinhold-Keller E, Herlyn K, Wagner-Bastmeyer R, Gross WL. Stable incidence of primary systemic vasculitides over five years: results from the German vasculitis register. Arthritis Rheum. 2005;53:93-9.

61. Centers for Disease Control and Prevention National Center for Health Statistics (NCHS). Underlying cause of death 1999-2016 on CDC WONDER online database. 2019. http://wonder.cdc.gov/ ucd-icd10.html. Accessed 14 Jun 2019. 
62. Statistics Canada (STATCAN). Deaths and mortality rates, by age group. Table: 13-10-0710-01. 2020. https://www150.statcan.gc. $\mathrm{ca} / \mathrm{t} 1 / \mathrm{tbl1} / \mathrm{en} / \mathrm{tv}$.action?pid=1310071001. Accessed $10 \mathrm{Jul} 2020$.

63. Federal Statistical Office (GBE). Statistics on the causes of death and state of the population. Table Deaths, Mortality figures (from 1998). 2020. http://www.gbe-bund.de/gbe10/trecherche .prc_them_rech?tk $=3600 \&$ tk $2=3800 \& p \_u i d=$ gast\&p_aid $=37561$ 247\&p_sprache $=E \& c n t \_u t=7 \& u t=3900$. Accessed 10 Jul 2020. 